\title{
Genetic diversity of a widespread annual killifish from coastal Tanzania
}

\author{
Veronika Bartáková', Béla Nagy², Matej Polačik', Radim Blažek', Hieromin Lamtane ${ }^{3}$ and Martin Reichard ${ }^{1 *}$ (D)
}

\begin{abstract}
Background: African annual killifishes (Nothobranchius spp.) are adapted to seasonally desiccating habitats (ephemeral pools), surviving dry periods as dormant eggs. Given their peculiar life history, geographic aspects of their diversity uniquely combine patterns typical for freshwater taxa (river basin structure and elevation gradient) and terrestrial animals (rivers acting as major dispersal barriers). However, our current knowledge on fine-scale interspecific and intra-specific genetic diversity of African annual fish is limited to a single, particularly dry region of their distribution (subtropical Mozambique). Using a widespread annual killifish from coastal Tanzania and Kenya, we tested whether the same pattern of genetic divergence pertains to a wet equatorial region in the centre of Nothobranchius distribution.

Results: In populations of Nothobranchius melanospilus species group across its range, we genotyped a part of mitochondrial cytochrome oxidase subunit 1 (COI) gene (83 individuals from 22 populations) and 10 nuclear microsatellite markers (251 individuals from 16 populations). We found five lineages with a clear phylogeographic structure but frequent secondary contact. Mitochondrial lineages were largely congruent with main population genetic clusters identified on microsatellite markers. In the upper Wami basin, populations are isolated as a putative Nothobranchius prognathus, but include also a population from a periphery of the middle Ruvu basin. Other four lineages (including putative Nothobranchius kwalensis) coexisted in secondary contact zones, but possessed clear spatial pattern. Main river channels did not form apparent barriers to dispersal. The most widespread lineage had strong signal of recent population expansion.

Conclusions: We conclude that dispersal of a Nothobranchius species from a wet part of the genus distribution (tropical lowland) is not constrained by main river channels and closely related lineages frequently coexist in secondary contact zones. We also demonstrate contemporary connection between the Ruvu and Rufiji river basins. Our data do not provide genetic support for existence of recently described cryptic species from N. melanospilus complex, but cannot resolve this issue.
\end{abstract}

Keywords: Dispersal, Eastern Africa, River morphology, Temporary pool, mtDNA, Historical demography

\section{Background}

Ephemeral habitats with patchy distribution are common but represent challenging environment to dispersal and colonization. While some species have evolved specialised stage to overcome dispersal limitation, others have responded by strong population resilience and possess stages resistant to shifting environmental conditions. For example, small cladocerans often produce resting stages that are dispersed by wind and,

\footnotetext{
* Correspondence: reichard@ivb.cz

${ }^{1}$ Czech Academy of Sciences, Institute of Vertebrate Biology, Květná 8, 60365 Brno, Czech Republic

Full list of author information is available at the end of the article
}

consequently, many cladoceran species have very large ranges and weak genetic structure (e.g. [1]). In contrast, stream gammarids (Amphipoda) are poor dispersers but resistant to temporary environmental challenges, and evolved into locally endemic lineages and species (e.g. [2]).

Temporary freshwater pools are common in highly seasonal environments, including African savanna. Their specialised fauna includes widespread invertebrate species with specific dispersal stage (e.g. crustaceans and aquatic insects) as well as specialised killifish species that are extremely poor dispersers [3, 4]. In African savanna, ephemeral pools inhabited by fishes vary in their 
temporal dynamics, from exclusively rain-fed pools of a brief existence ( $<1$ month) in semi-arid Mozambican savanna to semi-permanent networks of pools within active river alluvia in equatorial regions [5]. However, nothing is known on how such contrasting conditions affects connectivity of populations and their genetic diversity and phylogeographic structure.

Annual killifish are adapted to ephemeral pools in Africa and Neotropics by possessing a resilient developmental stage. After habitat desiccation, their embryos survive in dry pool sediment in the form of diapausing eggs $[6,7]$. Within the order Cyprinodontiformes (killifishes, toothcarps and livebearers) annual life history evolved at least six times [8], with habitat desiccation often becoming obligatory for successful embryo development [9]. In Eastern Africa, over 85 recognized species of the genus Nothobranchius inhabit ephemeral freshwater pools developed on vertisol (dark cracking clay) soils [5]. Nothobranchius distribution covers extensive region from relatively dry subtropical areas with a single rainy season in the north and south across humid equatorial areas with two rainy seasons a year. Nothobranchius diversification follows allopatric scenario [10], with isolating populations through separations of drainages through landscape faulting and warping [11, 12]. The same mode of diversification apparently pertains to intra-specific level. The dry southern part of Nothobranchius range (Mozambique) harbours highly geographically structured populations, with important roles of genetic drift and dispersal limitation [13-15]. In that region, major rivers formed significant barriers to killifish dispersal, leading to suture zones shared by coexisting Nothobranchius lineages [15]. In addition, intra-specific variation is structured along elevational gradient [15]. Such phylogeographic pattern is exceptional as it combines features of both aquatic and terrestrial taxa.

In the present study, we tested whether the patterns of Nothobranchius genetic diversity from dry subtropical region pertain to wet equatorial region of African savanna. Specifically, we investigated population genetic pattern in Nothobranchius melanospilus species group, geographically widespread and locally common killifish in lowland East Africa [16]. This species group contains Nothobranchius melanospilus (Pfeffer, 1896) and two recently described cryptic species; Nothobranchius prognathus Costa 2019 and Nothobranchius kwalensis Costa 2019 from peripheral parts of the N. melanospilus range [17]. The species group inhabits large lowland region of southeastern Kenya and eastern Tanzania, an important hotspot of biological diversity with a high occurrence of endemic species $[18,19]$, including notable diversity of Nothobranchius fishes [16]. Using 264 individuals from 22 populations, we combined information from mitochondrial cytochrome oxidase subunit $1(\mathrm{COI})$ gene and
10 nuclear microsatellite markers to examine the role of river basins, river channels and elevational gradient in structuring $N$. melanospilus species group.

\section{Methods}

Study taxon and study area

Nothobranchius melanospilus is the most commonly recorded species of the genus. It is found in natural temporary pools, swamps and small temporary streams as well as in ricefields, man-made ditches and culverts [16]. The species group has wide geographical distribution and its populations are common across coastal area $(3 \mathrm{~m}$ above sea level) up to elevation of $425-490 \mathrm{~m}$ in the upper Wami basin (Tendigo swamp) $[16,17]$. Geographically, populations are recorded from the Umba and Ramisi basins in the southeastern tip of Kenya, across large region of coastal Tanzania (Pangani, Wami, Ruvu, Mbezi, Ruhoi and Rufiji basins) and from the island of Zanzibar off the Ruvu river [16, 20, 21].

A recent taxonomic work used museum specimens [17] to formally describe existence of two previously unrecognised species within $N$. melanospilus, on the basis of combination of morphometric characters and female colouration. Based on that study [17], N. melanospilus sensu stricto is distributed only south of the Wami basin (and on Zanzibar Island). The upper Wami basin populations were described as Nothobranchius prognathus Costa 2019 and populations from southeastern Kenya as Nothobranchius kwalensis Costa 2019. Hence, our study putatively concerns to a complex of three closely related species of the $N$. melanospilus group. Our samples cover the entire range of the species group, except for Zanzibar Island and the lower Pangani basin.

The region of $N$. melanospilus species group distribution was modified by East African Rift tectonics that formed the Ruvu and Rufiji throughs [5]. The two basins share a common swampy area in their middle and upper reaches. Island of Zanzibar, also inhabited by $N$. melanospilus species, is located on a shallow shelf near the mouths of the Ruvu and Wami rivers and was likely linked to mainland populations until 12,000 years ago. Small coastal rivers between the Ruvu and Rufiji (e.g. the Luhule and Mbezi rivers) are separated from the Ruvu and Rufiji by Pugu and Mtoti hills, forming so-called Mbezi Triangle with endemic Nothobranchius species $[5,16]$. The lower reaches of Wami and Pangani and a small Ramisi river share a low-lying coastal strip, while the upper Wami basin (Tendigo swamp) has limited connection to the floodplain pools of the lower Wami basin [5].

\section{Sampling and genotyping}

Specimens from most populations were collected during a dedicated field trip in May and June 2017, 
using dip and seine nets. Fish were identified in the field; unlike in other Tanzanian Nothobranchius, female $N$. melanospilus species group are readily recognised from other Nothobranchius species by their unique dark spots on the body [16]. Most fish were identified on the bank, small fin clips were taken from their caudal fin and stored in 98\% ethanol. Fish were then released back to their habitat. Voucher specimens (a random subsample of both sexes) were taken from most populations and are stored at the Institute of Vertebrate Biology, Brno, Czech Republic. Sixteen specimens from 7 populations were collected by B.N. (Table 1), using a similar method. This included a sample of putative N. kwalensis from the Ramisi basin (4 individuals from 2 populations). All field sampling and export procedures followed regulations of Tanzania, with permits and research associateship issued by Sokoine University of Agriculture in Morogoro (research permit: RPGS/R/AS/11/ 2017; export permit AS/A/1).

In the laboratory, DNA was extracted using the DNeasy Blood and Tissue Kit (Qiagen) following a standard protocol. Full details of the genotyping methods, primer sequences, microsatellite multiplexing, and PCR protocols are presented in Additional file 1. In brief, partial mitochondrial COI gene was amplified using primers TRNYF1 (AGG GAG TTA CAA TCC ACC ACT ATT T) and TRNSR1 (ATG GGG GTT CAA TTC CTT CCT TT), alternatively, and a forward primer COI852F (CTT TAT TGT TTG AGC CCA CCA CA) [12] for a set of 83 individuals from 22 populations (Table 1). PCR products were sequenced commercially in Macrogen and GATC Biogen. All sequences have been deposited in GenBank (accession numbers MN413245-MN413327). Initially, we aimed to genotype partial cytochrome $b$ gene, but none of the 7 tested primers (Additional file 1) amplified successfully.

We used a set of 10 microsatellite loci in four multiplex PCR sets (for details see Additional file 1) to genotype a sample of the 251 individuals from 16 populations (Table 1). PCR products were separated on the ABI Prism ${ }^{\circ}$ 3130 Genetic Analyzer (Applied Biosystems) and analysed using GeneMapper v. 3.7 (Applied Biosystems).

Table 1 Overview of analysed populations, with their collection code (Population ID), GPS coordinates, identification of river basin, elevation (in $\mathrm{m}$ above sea level), number of individuals analysed on 10 microsatellite markers ( $\mathrm{N}_{\mathrm{MS}}$ ) and on mitochondrial COI sequence $\left(\mathrm{N}_{\mathrm{CO}}\right)$, and assignment of individuals to one of five haplogroups (Haplogroup). Populations are ranked by their elevation within river basins

\begin{tabular}{|c|c|c|c|c|c|c|c|c|}
\hline Population ID & GPS_S & GPS_E & Basin & Elevation & Habitat type & $\mathrm{N}_{\mathrm{MS}}$ & $\mathrm{N}_{\mathrm{COI}}$ & Haplogroup \\
\hline T15 & 6.63624 & 38.16554 & Ruvu & 246 & Isolated pool & 21 & 4 & Wami \\
\hline T14 & 6.86204 & 38.18471 & Ruvu & 161 & Ephemeral stream & 17 & 4 & Ruvu \\
\hline T62 & 6.60366 & 38.33852 & Ruvu & 166 & Pool & 2 & 3 & Ruvu \\
\hline TZN 09-2a & 6.69247 & 38.75305 & Ruvu & 62 & Pool & 0 & 3 & Ruvu \\
\hline T57 & 6.69268 & 38.75316 & Ruvu & 62 & Pool & 27 & 4 & Ruvu \\
\hline T02 & 6.70380 & 38.67541 & Ruvu & 22 & Pool & 29 & 3 & Ruvu \\
\hline T64 & 6.46973 & 38.79884 & Ruvu & 21 & Man-made pools & 19 & 4 & Ruvu \\
\hline TZN 09-1 & 6.46063 & 38.90732 & Ruvu & 19 & Pool & 0 & 3 & Ruvu/Mbezi \\
\hline T51 & 6.45595 & 38.90742 & Ruvu & 17 & Rice field & 3 & 3 & Ruvu \\
\hline T50 & 6.51363 & 38.95730 & Ruvu & 14 & Pool & 9 & 6 & Mbezi \\
\hline TZN 17-9 & 6.47548 & 38.85812 & Ruvu & 4 & Floodplain pool & 12 & 3 & Ruvu \\
\hline T17 & 8.12097 & 38.96849 & Rufiji & 50 & Pool & 9 & 6 & Ruvu \\
\hline TZN $18-2^{a}$ & 8.10159 & 38.99509 & Rufiji & 30 & Pool & 0 & 1 & Rufiji \\
\hline T16 & 8.07289 & 38.98788 & Rufiji & 23 & Culvert by main road & 18 & 7 & Rufiji/Ruvu \\
\hline TZN $17-1^{a}$ & 8.05565 & 38.98293 & Rufiji & 20 & Pool & 0 & 2 & Rufiji/Ruvu \\
\hline T31 & 7.19349 & 39.17192 & Mbezi & 65 & Deep pool with rice field & 22 & 4 & Mbezi \\
\hline T35 & 7.35934 & 39.12495 & Mbezi & 31 & Rice field & 20 & 8 & Ruvu \\
\hline KEN $15-1^{a}$ & 4.52267 & 39.29908 & Ramisi & 21 & Floodplain pool & 0 & 1 & Ramisi \\
\hline KEN 08-23a & 4.51842 & 39.29303 & Ramisi & 21 & Pool & 0 & 3 & Ruvu \\
\hline T06 & 6.59145 & 37.59217 & Wami & 435 & Swamp & 21 & 4 & Wami \\
\hline T09 & 6.72178 & 37.12161 & Wami & 425 & Pool in swampy area & 19 & 4 & Wami \\
\hline T83 & 6.76608 & 37.16220 & Wami & 425 & Pool & 3 & 3 & Wami \\
\hline
\end{tabular}

${ }^{\mathrm{a}}$ samples collected by Béla Nagy 


\section{Analysis of mitochondrial DNA variation and historical demography}

Phylogenetic relationships within COI dataset were inferred by Bayesian (BI) approach. We used PartitionFinder v. 2.1.1 [22] to select the most suitable substitution models for different parts of mtDNA using the corrected Akaike Information Criterion (HKY, GTR, and SYM + I for individual positions in codon). One sequence of Nothobranchius guentheri was used as an outgroup. Bayesian analysis was performed by Markov Chain Monte Carlo (MCMC) simulation using MrBayes 3.2.6 [23]. Two independent analyses were initiated from random trees. Three heated and one cold chain were run for 20 million generations per run, sampling every 1000 generations, and 25\% of trees were discarded as burn-in. Bayesian posterior probabilities were used to evaluate branch support of the tree. Phylogenetic analysis was performed on Cipres Science Gateway webserver [24] and the final tree was edited in FigTree v1.3.1 (http://tree.bio.ed. ac.uk/software/figtree).

All sequences were geo-referenced and the geographical distribution of lineages was plotted onto map using QGIS 2.18 (http://qgis.org). Diversity estimates, i.e. number of polymorphic sites $(N p)$, number of haplotypes $(N h)$, haplotype diversity $(H d)$, nucleotide diversity $(\pi$, expressed as percentages, i.e. $0.001=0.1 \%$ ), the average number of nucleotide differences $(k)$ and Watterson's estimate of $\theta\left(\theta=4 \mathrm{Ne}^{*} \mu\right)$ were calculated using DnaSP v. 5.10 .01 [25].

Within two most widespread lineages, historical demography was estimated using the neutrality tests, Tajima's D and Fu's FS, sensitive to population size changes [26] in DnaSP [25], with significantly negative values reflecting recent population expansion. Ramos-Onzins and Rozas R2 tests were also computed due to relatively lower sample size, with $P$-values obtained by coalescent simulations with 10,000 replicates in DnaSP [25]. Additionally, the distribution of pairwise nucleotide differences (mismatch distribution; MD) was calculated in DnaSP. We used the sum of square deviations (SSD) between the observed and expected mismatch as a test statistic for the validity of the estimated stepwise expansion model [27]. Parameter $\tau$ (the moment estimator of time to the expansion) was estimated with DnaSP using the moment method of Rogers [28] assuming the infinite sites model (IFM) and, additionally, in ARLEQUIN [29] using the method of Schneider and Excoffier [27] to relax the IFM assumption. Confidence intervals were obtained by a parametric bootstrap approach based on 1000 replicates performed in ARLEQUIN [29].

\section{Intra-population analysis of microsatellite marker variation}

The proportion of null alleles (NA) at each locus and population was estimated in FreeNA [30]. The mean frequency of microsatellite null alleles per population was greater than $5 \%$ for five loci (Additional file 1). The greatest proportion of null alleles was $13.6 \%$ for $\mathrm{Nfu}_{-}$ 0027_FLI locus.

Deviations from linkage and Hardy-Weinberg equilibrium (HWE) for each locus and population were detected in Genepop 4.0.10 [31, 32]. Linkage disequilibrium among 10 microsatellite loci and HWE ("Exact probability test") were tested using Markov chain methods (dememorization: 10,000, batches: 100, iterations per batch: 5000). Correction for multiple testing was performed using false discovery rate approach (FDR) in QVALUE [33]. Only 5 out of 441 pair-wise results of genotypic linkage disequilibrium tests were significant at $p<0.05$. Pairs of loci were significantly linked only in one or two populations and the microsatellite loci can be considered to be unlinked.

Genetic variability was estimated by calculating observed heterozygosity $\left(\mathrm{H}_{\mathrm{O}}\right)$ and unbiased expected heterozygosity according to Nei (1978) $\left(\mathrm{H}_{\mathrm{E}}\right)$ in GENETIX 4.05.2 [34]. Mean allelic richness (AR) was determined with the rarefaction method in FSTAT 2.9.3 [35] to estimate the expected number of alleles standardized to the smallest population sample of 8 individuals. Pairwise genetic differentiation was calculated with GENETIX 4.05.2. For analysis of genetic variability $\left(\mathrm{HWE}, \mathrm{H}_{\mathrm{O}}, \mathrm{H}_{\mathrm{E}}\right.$, AR) populations with $<8$ sampled individuals were not used (thus excluding populations T51, T62, and T83).

\section{Inter-population analysis of genetic structure}

To quantify genetic differentiation between populations, we computed pairwise estimators of $F_{\mathrm{ST}}$ for each pair of populations using the ENA correction described in [30] and implemented in the software FreeNA [30], as there was some evidence of null alleles. We then used these corrected values to test for isolation-by-distance pattern by regressing pairwise estimates of $F_{\mathrm{ST}} /\left(1-F_{\mathrm{ST}}\right)$ against ln-distance between sample sites. Mantel tests were used to test the correlation between matrices of genetic differentiation and geographical distances between sampling sites by 1000 permutations in Genepop 4.0.10 [32].

To investigate the spatial genetic structure among individuals, we used STRUCTURE 2.3.4 [36]. The individualbased Bayesian clustering procedure was run with 20 independent runs for each of $\mathrm{K}$ from 1 to 10 . Each run included $10^{6}$ iterations, following a burn-in period of $10^{5}$ iterations. We used admixture ancestry model and correlated allele frequencies model (with $\lambda=1$ ). The output of STRUCTURE analysis was post-processed in CLUMPAK software [37] to identify separate groups of runs on the base of similarity between Q-matrices for each K. We used the LargeKGreedy algorithm, random input order and 2000 repeats. Different modes from the results of the 20 runs for each $\mathrm{K}$ value at a threshold of 0.9 for similarity scores were identified. Summary barplots for a given $\mathrm{K}$ value contain 
averaged proportions of individual membership obtained for all runs in the same mode. The likelihood of K ( $\mathrm{Ln}$ $\operatorname{Pr}(\mathrm{X} \mid \mathrm{K}))$, the $\Delta \mathrm{K}$ criterion [38] and a proportion of similar runs that formed the major modes for each $\mathrm{K}$ were used to infer the best number of real populations.

\section{Results}

Mitochondrial lineages: distribution and demographic history

Among 22 populations (83 individuals), we detected 30 different haplotypes of $C O I$ sequences $(657 \mathrm{bp})$. Their phylogenetic analysis revealed five main lineages, some of which possessed a finer substructure (Fig. 1, Additional file 2). The lineages had clear geographic structure but common contact zones (Fig. 2) and generally received low statistical support (Fig. 1). Only the basal lineage (Rufiji, red in Fig. 2) had strong node support (Fig. 1). This lineage was found exclusively in the Rufiji basin, in the south of the species range, where it widely coexisted with the second lineage (Ruvu, blue). The Ruvu lineage was most common and geographically most widespread. It was dominant in the middle and lower Ruvu basin, but found across the north-south axis of the species range, from coastal Kenya to the Rufiji basin, including Mbezi Triangle. Small coastal basins of Mkuza and Mbezi harboured individuals from the third lineage (Mbezi, green) that locally coexisted with the Ruvu lineage in the lower Ruvu basin (population T91). The putative $N$. prognathus was represented by the fourth lineage (Wami, orange), from pools within the upper Wami River basin (Tendigo swamp). In addition, Wami lineage pertained to one pool in an isolated part of the middle Ruvu basin (population T15). This haplogroup was distributed at the highest elevation (246435 masl compared to $<167$ masl in other lineages), but had low statistical support $(\mathrm{BI}=0.76$, Fig. 1$)$. Finally, a single individual with a unique haplotype (Ramisi, yellow lineage) was found in coastal Kenya (population KEN08-23), perhaps the putative Nothobranchius kwalensis. More specimens were not available from that population. The distinctness of this haplotype had low statistical support $(\mathrm{BI}=0.65$, Fig. 1$)$, though we note that our phylogenetic inference is based only on a fragment of $657 \mathrm{bp}$. Interestingly, all three individuals from the adjacent population (KEN15-1, located only $15 \mathrm{~km}$ from KEN08-23) possessed haplotypes of the common Ruvu lineage (Fig. 2).

Demographic history was analysed separately for geographically two most widespread mitochondrial lineages - Ruvu (blue) and Wami (orange). Unimodal pattern of mismatch distribution graphs (Additional file 2) and Ramos-Onsins \& Rozas' R2 (Table 2) suggested recent population expansion of both haplogroups. The tests of neutrality (Tajima's D and Fu's FS) sensitive to sample size limitations demonstrated significant expansion only in the Ruvu haplogroup (Table 2). The sums of squared deviations (SSD) of the mismatch distribution were not significant, indicating that the curves support the sudden expansion model. Full details of $\mathrm{COI}$ variability and historical demography are shown in Table 2.

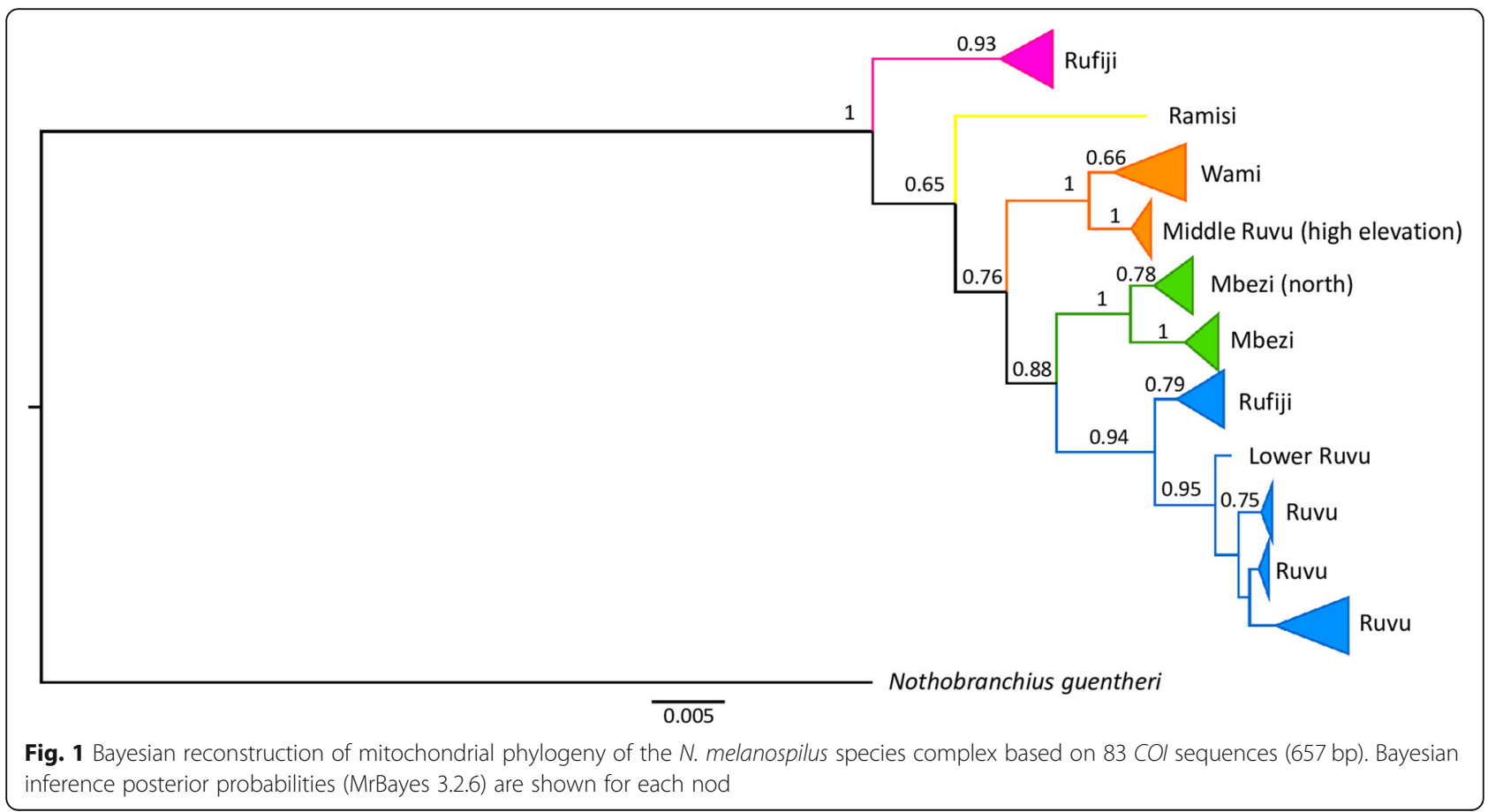




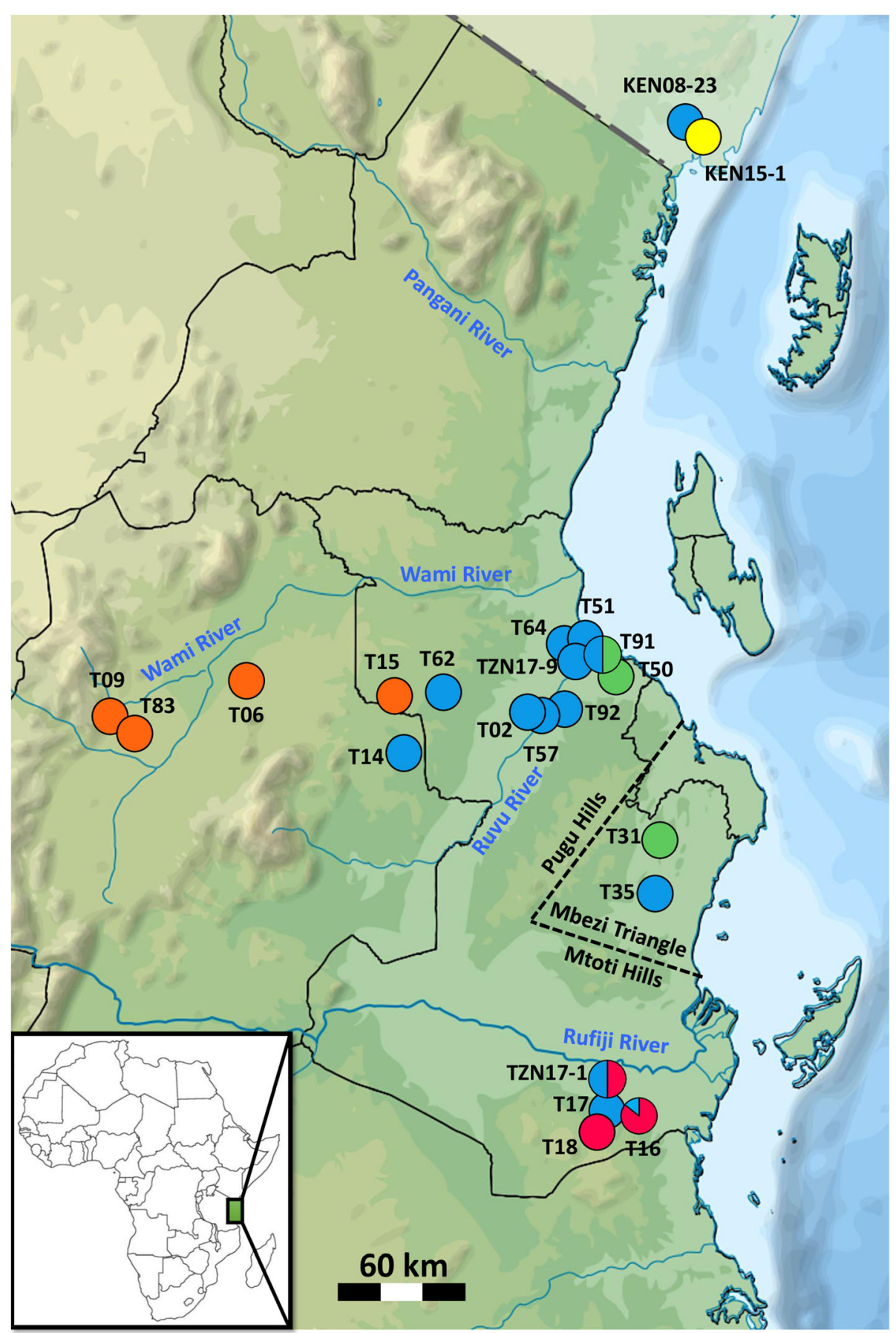

Fig. 2 Geographic distribution of Nothobranchius melanospilus-species group mitochondrial lineages. The colours correspond to the lineages as defined in Fig. 1 and indicate the relative proportions of lineages at a particular locality. Names of localities correspond to those in Table 1. The map has been modified from open-access source map that is free to re-use and adapt under CC-BY-SA-3.0 licence and is available at https://commons.wikimedia.org/wiki/File:Tanzania_relief_location_map.sv

\section{Nuclear microsatellite markers: intra-population} variability

Based on data from 10 microsatellite loci, most populations (nine out of 13) showed deviance from HWE, when calculated over all loci (Table 3). In most cases deviations from HWE were caused by null alleles present with an increased frequency at some loci and populations, probably as a result of the "ascertainment bias".

All measures of intra-population genetic variation $\left(\mathrm{H}_{\mathrm{O}}, \mathrm{H}_{\mathrm{E}}, \mathrm{AR}\right)$ for populations with at least 8 individuals 
Table 2 Analysis of mitochondrial variability and historical demography. The number of sequences (N), number of haplotypes (h), haplotype (gene) diversity with one Standard Deviation (Hd), number of polymorphic (segregating) sites (S), nucleotide diversity (in \%; with one Standard Deviation) ( $\pi$ ), average number of nucleotide differences (k), Tajima's D (with significance at $P<0.05$ denoted by asterisk), Fu's FS (with significance at $P<0.01$ denoted by asterisk), Ramos-Onsins and Rozas R2 (with significance at $P<0.05$ denoted by asterisk), onset of population expansion assuming the stepwise growth model ( $\tau$ Arl, with 95\% confidence interval), $\tau$ DnaSP (the moment estimator of time to the expansion), sum of squared deviations (SSD)and the probability of observing a less good fit between the model and the observed distribution by chance $\left(P_{S S D}\right)$ and the mismatch observed mean (ObsMean)

\begin{tabular}{|c|c|c|c|c|c|c|c|c|c|c|c|c|c|c|}
\hline Haplogroup & $\mathrm{N}$ & $\mathrm{h}$ & $\mathrm{Hd}$ & $S$ & $\pi(\%)$ & k & Tajima's D & $\begin{array}{l}\text { Fu's } \\
\text { FS }\end{array}$ & R2 & т Arl $(95 \% \mathrm{Cl})$ & т DnaSP & SSD & $P_{S S D}$ & ObsMean \\
\hline All pooled & 83 & 30 & $0.858 \pm 0.035$ & 67 & $1.909 \pm 0.147$ & 12.525 & - & - & & - & - & - & - & - \\
\hline Wami & 15 & 9 & $0.886 \pm 0.062$ & 11 & $0.403 \pm 0.057$ & 2.648 & -0.838 & -3.30 & $0.050^{*}$ & $3.334(0.842-5.777)$ & 2.648 & 0.00887 & 0.589 & 2.648 \\
\hline Ruvu & 44 & 11 & $0.593 \pm 0.087$ & 13 & $0.148 \pm 0.038$ & 0.973 & $-2.068^{*}$ & $-7.41 *$ & $0.101^{*}$ & $0.859(0.391-1.563)$ & 0.252 & 0.00268 & 0.545 & 0.973 \\
\hline
\end{tabular}

Note that recently admixed populations were excluded from analyses

are shown in Table 3. The range of AR was 4.60-8.62 (rarefaction estimate for the lowest sample size $N=8$ ). The lowest intra-population genetic variability $\left(\mathrm{H}_{\mathrm{E}}<\right.$ $0.70, \mathrm{AR}<5.2$ ) was detected in populations $\mathrm{T} 57, \mathrm{~T} 31$ and T15 located at relatively isolated pools. In contrast, the highest intra-population genetic diversity $\left(\mathrm{H}_{\mathrm{E}} \geq 0.85\right.$, $A R$ > 7.9) was found in populations from the floodplain of lower parts of the major rivers (Ruvu: TZN17-9, T64, T02, all below 22 masl; Rufiji: T16, T17, below 50 masl) and in population T14 in the middle reach of the Ruvu (Table 3).

\section{Nuclear microsatellite markers: genetic structure}

We detected high level of genetic structuring among $N$. melanospilus-group populations, with the mean ( \pm S.E.) pairwise $F_{\mathrm{ST}}=0.113 \pm 0.07$. The pairwise $F_{\mathrm{ST}}$ values were significantly different from zero in $95.83 \%$ of population pairs; only five of 120 pairwise $F_{\mathrm{ST}}$ were not significant (Additional file 3). Four non-significant $F_{\mathrm{ST}}$ values were between pairs of geographically close populations, though one non-significant $F_{\mathrm{ST}}$ was between geographically distant populations from different basins (T14 and T17, from the Ruvu and Rufiji basins, respectively). The pattern of isolation-by-distance showed weak but significant association between geographical and genetic distances (Mantel test, 1000 permutations, $P=0.03$, Additional file 2: Figure S2).

Using Bayesian clustering in STRUCTURE, the most suitable model to separate sampled populations was for $K=8$, based on the likelihood of $\mathrm{K}(\operatorname{Ln} \operatorname{Pr}(\mathrm{X} \mid \mathrm{K}))$, the $\Delta \mathrm{K}$ criterion [38], and a proportion of similar runs (Additional file 4, Fig. 3). The Wami basin populations were consistently separated from all other populations (Fig. 3). Populations from the Ruvu and Rufiji basins, as well as populations from Mbezi Triangle were all clustered at lower $K$ values, but increasing the number of assumed clusters led to the separation of two Mbezi populations (T31, T35) to reciprocally unique clusters (Fig. 3). Three individual populations were

Table 3 Measures of intra-population genetic variability based on analyses of microsatellite markers. Sample size (N), P-values of the Fisher's exact test for deviation from Hardy-Weinberg equilibrium (HWE), expected heterozygosity based on Nei estimate ( $\left.H_{E}\right)$, observed heterozygosity $(\mathrm{HO})$ and allelic richness estimated for 8 individuals using rarefaction (AR). Populations are ranked from highest AR

\begin{tabular}{|c|c|c|c|c|c|c|}
\hline Population & Basin & $\mathrm{N}$ & HWE & $\mathrm{H}_{\mathrm{E}}(\mathrm{Nei})$ & $\mathrm{H}_{\mathrm{O}}$ & AR \\
\hline T14 & Ruvu & 17 & $<0.001$ & $0.8796 \pm 0.0693$ & $0.7381 \pm 0.1881$ & 8.615 \\
\hline T64 & Ruvu & 19 & $<0.001$ & $0.8580 \pm 0.0916$ & $0.7269 \pm 0.2265$ & 8.363 \\
\hline T02 & Ruvu & 29 & $<0.001$ & $0.8531 \pm 0.0865$ & $0.6823 \pm 0.2008$ & 8.235 \\
\hline TZN 17-9 & Ruvu & 12 & $<0.001$ & $0.8497 \pm 0.1095$ & $0.7203 \pm 0.1847$ & 8.181 \\
\hline $\mathrm{T} 17$ & Rufiji & 9 & $<0.001$ & $0.8458 \pm 0.1071$ & $0.6458 \pm 0.3079$ & 7.939 \\
\hline T16 & Rufiji & 18 & $<0.001$ & $0.8565 \pm 0.0807$ & $0.7233 \pm 0.2086$ & 7.934 \\
\hline T09 & Wami & 19 & 0.0217 & $0.7331 \pm 0.3114$ & $0.6737 \pm 0.3391$ & 7.819 \\
\hline T50 & Ruvu & 9 & $<0.001$ & $0.8437 \pm 0.1040$ & $0.5944 \pm 0.3248$ & 7.643 \\
\hline Т35 & Mbezi & 20 & $<0.001$ & $0.8270 \pm 0.1252$ & $0.6611 \pm 0.1788$ & 7.260 \\
\hline T06 & Wami & 21 & 0.2836 & $0.6907 \pm 0.3046$ & $0.6652 \pm 0.3290$ & 6.504 \\
\hline Т15 & Ruvu & 21 & 0.0083 & $0.6920 \pm 0.1694$ & $0.6375 \pm 0.2992$ & 5.162 \\
\hline T31 & Mbezi & 22 & 0.1695 & $0.6339 \pm 0.2561$ & $0.6089 \pm 0.2904$ & 4.716 \\
\hline T57 & Ruvu & 27 & $<0.001$ & $0.6785 \pm 0.1736$ & $0.5362 \pm 0.2323$ & 4.600 \\
\hline
\end{tabular}




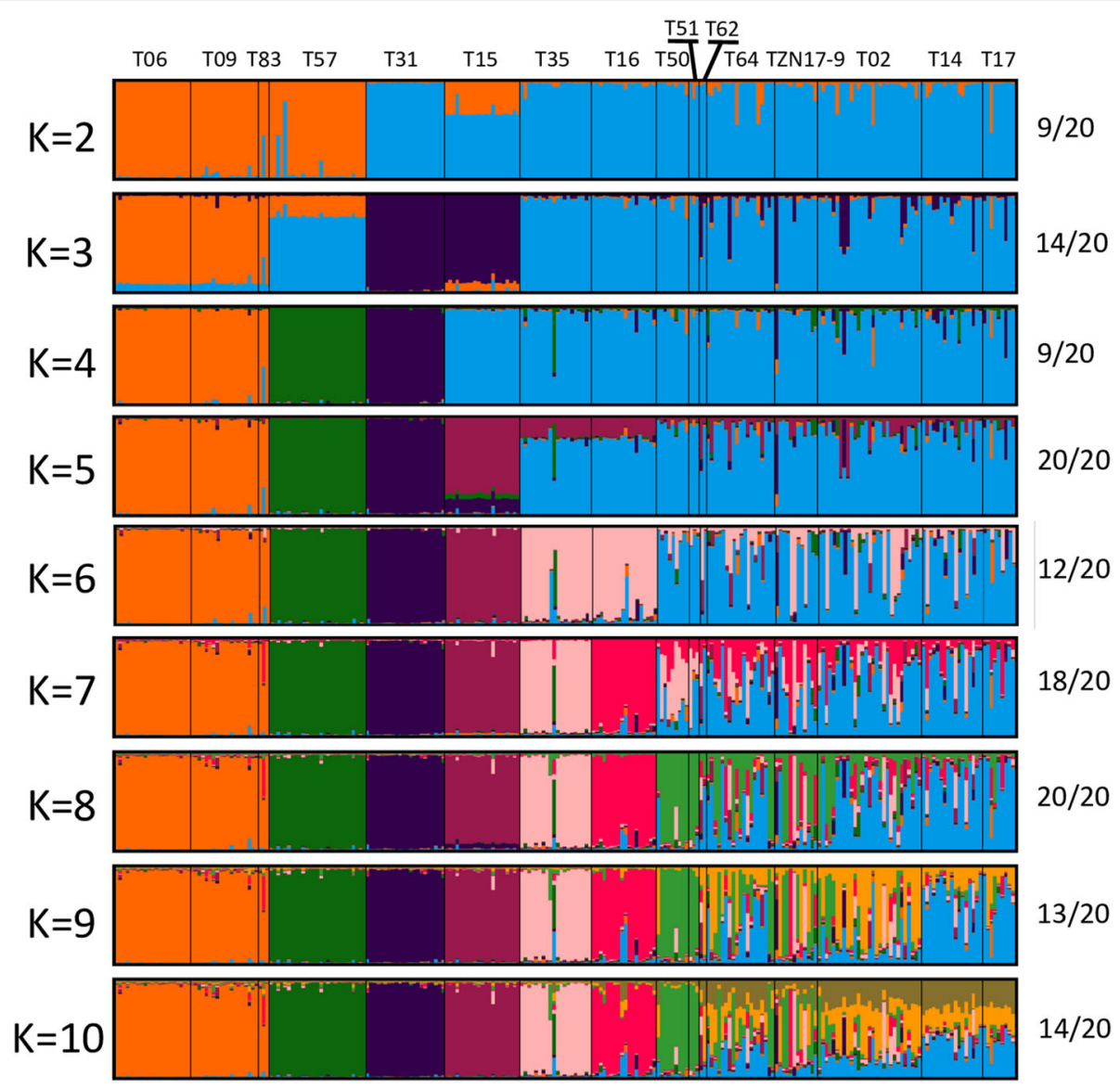

Fig. 3 Bayesian analysis of genetic similarity among Nothobranchius melanospilus-species group populations performed in STRUCTURE for 251 individuals from 16 populations for $K=2-10$

separated to unique clusters at $K=8$; isolated populations T15 (from the Wami lineage) and T57 (Ruvu lineage) from the Ruvu basin (both with low intra-population variability), and T16 (one fish from the Ruvu and six fish from the Rufiji lineage) from the Rufiji basin (Fig. 4). Finally, one lower Ruvu (T51, Ruvu lineage) and one lower Mkuza (T50, Mbezi lineage) populations, both located very close to the coast clustered together at $\mathrm{K}=8$ (Fig. 4).

\section{Discussion}

Populations of Nothobranchius melanospilus species group were clearly structured, with geographically adjacent lineages sometimes coexisting within a single population. Clustering to mitochondrial lineages had a good correspondence in nuclear microsatellite data. The most widespread lineage (Ruvu, blue) was found in all sampled regions except of the Wami basin. It coexisted with Rufiji (red) lineage in the lower Rufiji basin and Mbezi (green) lineage in the lower Ruvu. Its broad distribution agrees with a clear signature of recent expansion indicated by neutrality indices. Despite their coexistence, the three common lineages (Ruvu, Rufiji, Mbezi) had their apparent centres of distribution that are indicative of potential refugial persistence in the lower Ruvu basin, lower Rufiji basin and smaller coastal rivers between the Ruvu and Rufiji, including Mbezi Triangle.

Three upper Wami basin populations formed a separate cluster on mitochondrial and nuclear markers, apparently consistent with a recent elevation of the populations from this region to a specific level, as $N$. prognathus [17]. In our mitochondrial dataset, this lineage additionally included one population (T15) from an isolated pool at the periphery of the Ruvu basin, but this population did not cluster with the three Wami populations on nuclear markers. On nuclear markers, this population was recovered as relatively unique since it also differed from geographically close Ruvu population. The difference was likely driven by its low intrapopulation genetic diversity and hence important contribution of genetic drift. Indeed, that isolated population is located at relatively high elevation (246 masl; compared to $\leq 166$ masl in all other non-Wami populations, 

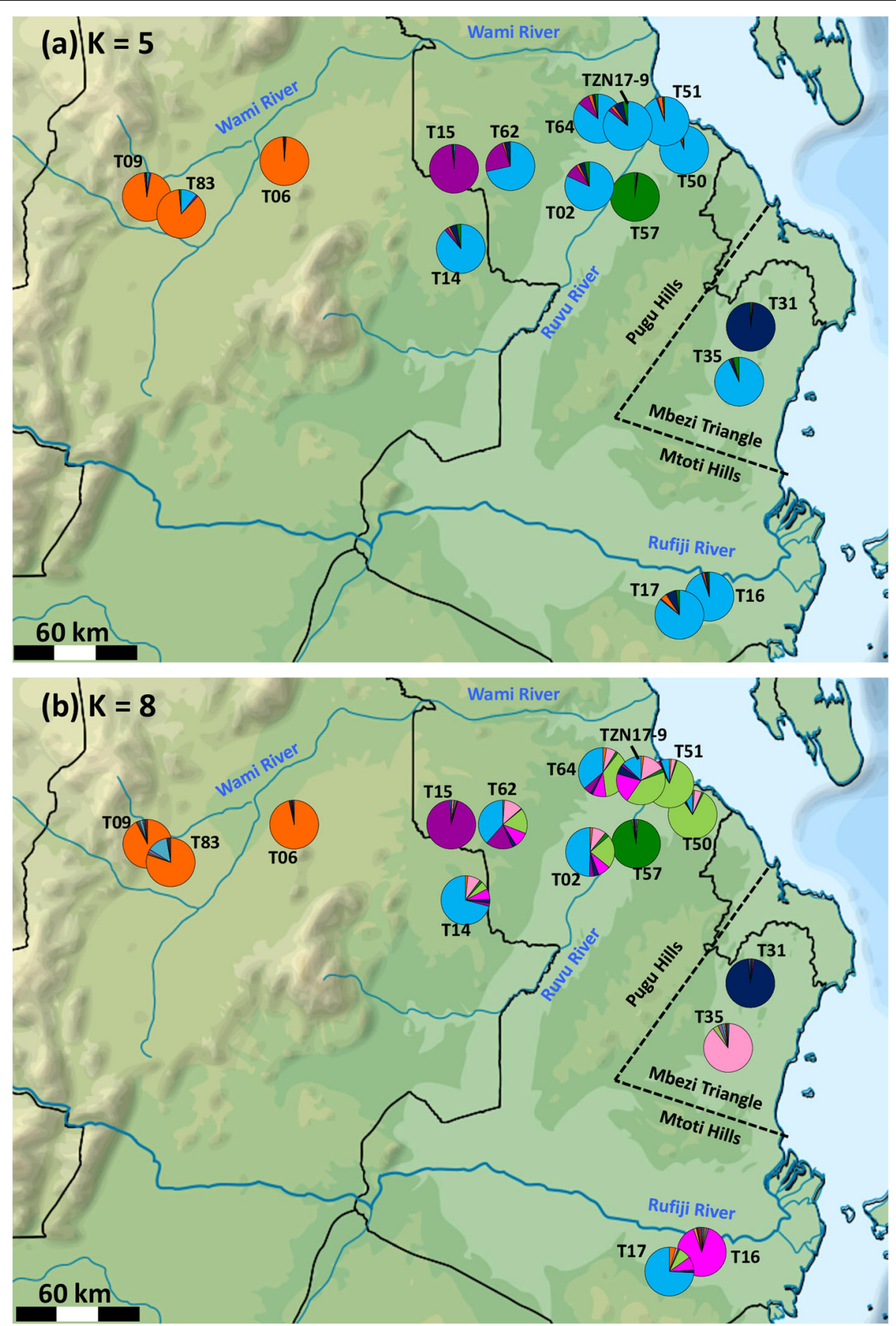

Fig. 4 Geographic distribution of genetic diversity in Nothobranchius melanospilus-species group from nuclear microsatellites based on assignment to 8 clusters (a) and 5 clusters (b) following STRUCTURE analysis across study area. Pie chart colours represent the proportional membership of individuals to microsatellite-based clusters inferred from the models selected using the approach of Evanno et al. [38]. Names of localities correspond to those in Table 1. The map has been modified from open-access source map that is free to re-use and adapt under CCBY-SA-3.0 licence and is available at https://commons.wikimedia.org/wiki/File:Tanzania_relief_location_map.svg

and 425-435 masl in the Wami populations). Unlike for the other four lineages, we have not confirmed coexistence of the Wami lineage with any other haplogroups in the sample of populations we studied. Investigation of finer-scale population genetic pattern in that region could resolve separation of the Wami and Ruvu lineages and clarify whether $N$. prognathus should be considered as a valid species.

A single divergent haplotype has been discovered in coastal Kenya (Ramisi basin, KEN15-1). Validity of this 
haplotype has been confirmed by repeated analysis (including new DNA sampling from voucher specimen). Morphological inspection of the voucher individual (adult male) confirmed that it belongs to $N$. melanospilus-species group. This region is terra typica of $\mathrm{N}$. kwalensis, a newly described cryptic species that can only be unambiguously diagnosed for female specimens [17], which were not available to us. While this apparently supports distinction of this lineage, a sample from adjacent population (KEN08-23, $15 \mathrm{~km}$ distant from KEN15-1 with no apparent dispersal barrier) contained three individuals with the widespread $N$. melanospilus haplotype (Ruvu) and morphologically, fish were typical $N$. melanospilus. Despite its relative distinctness, there was no statistical support to separate this haplotype. Clearly, more individuals and markers are needed to resolve validity of $N$. kwalensis. Our sampling (and laboratory analyses) were completed prior to the description of $N$. kwalensis and $N$. prognathus as separate species and we have no other specimens to further elaborate on our tentative findings. We acknowledge that this leaves the question of validity of $N$. kwalensis, and its geographical distribution, unresolved. Given the presence of the Ruvu lineage of $N$. melanospilus in coastal Kenya, it is possible that $N$. melanospilus sensu stricto rather than N. kwalensis may inhabit coastal plains of the northern Tanzania.

Regardless the taxonomic implications, we conclude that current $N$. melanospilus species group populations have been separated into at least five refugia that are consistent with divisions into the main river basins in the region. The lineage from at least one refugium (Ruvu) undergoes recent expansion and coexists with at least two other $N$. melanospilus lineages. Importantly, the only mitochondrial lineage (Rufiji) with a significant support for its genetic distinctiveness from other $N$. melanospilus-species-group lineages coexist widely with the Ruvu lineage and nuclear markers did not indicate the lack of panmixia. The fact that two lineages that were formally described to represent cryptic species of the complex - from the Wami (N. prognathus) and Ramisi (N. kwalensis) - are less distinct than the Rufiji lineage apparently supports the arguments of Wildekamp [39], who regarded minor morphological differences between $N$. melanospilus sensu stricto and the two putative cryptic species as normal intraspecific variation commonly seen in this [39] and many other Nothobranchius species [16, 40]. We acknowledge, however, that our results do not contradict existence of the two cryptic species either.

Intra-specific structure derived from microsatellite markers was largely congruent with mitochondrial data. Given a low number of individuals available, we have not genotyped Kenyan populations on microsatellite markers. Several populations that were not differentiated on mitochondrial data formed separate clusters at finer genetic substructuring. Those populations were typically genetically depauperated (low $H_{e}, H_{o}$ and $A R$ estimates), suggesting that their distinct population genetic signatures arose from genetic drift due to either population bottlenecks or founder effects.

The populations were principally structured by their respective river basins. Main channels of large rivers did not constitute apparent barriers to dispersal in N. melanospilus this species group, in contrast to annual killifishes in particularly dry regions of Africa [15]. For example, two populations inhabiting the opposite banks of the lower Ruvu (T64 and TZN 17-9) had negligible (and non-significant) $F_{\mathrm{ST}}$ value $(0.0042)$. Other adjacent populations had non-significant $F_{\mathrm{ST}}$ values, especially in the lower Ruvu (Additional file 3), but also in the Wami basin $(7 \mathrm{~km}$ distant T09 and T83 in Tendigo swamp; $\left.F_{\mathrm{ST}}=-0.012\right)$ and, unexpectedly, between Ruvu (T14) and Rufiji (T17) populations (distance $164 \mathrm{~km}, F_{\mathrm{ST}}=$ - 0.008). This corroborates that dispersal in equatorial region of coastal Tanzania might be more suitable for frequent dispersal across main river channel and among adjacent populations. The region experiences much longer duration of the wet phase, with two rainy seasons each year and a longer duration of habitat inundation compared to a single rainy season in subtropical Mozambique [41] with very brief periods of inundation $[42,43]$. It demonstrates that Nothobranchius fishes experience variable climatic and ecological challenges that may affect their dispersal, diversification and coexistence in local killifish assemblages [44].

Our data on Nothobranchius melanospilus species group are largely congruent with phylogeographic patterns of other cyprinodontid fishes. For example, Rivulus cylindraceus from Cuba has wide distribution, with two haplogroups coexisting in some adjacent drainages and highly divergent haplogroups in isolated edges of the range [45]. In Fundulus olivaceus from midwestern and southern USA, distinctive haplogroups coexist in secondary contacts across drainages, while in sympatric Fundulus notatus four mitochondrial haplogroups are strictly isolated by respective river basins [46]. Finally, geographically widespread mummichog killifish, Fundulus heteroclitus from Atlantic coast of North America combines latitudinal isolation-by-distance pattern with a division into two sharply separated clades [47].

Within annual killifish, genetic variability of Neotropical annual killifish from the genus Austrolebias is also not structured by main river channels [46]. Especially lowland parts of major basins have been strongly affected by repeated marine transgressions and regressions during late Pliocene and Pleistocene [48]. For example, Austrolebias bellottii species group is widespread throughout the lower Paraná/La Plata and Uruguay basins, in a situation very similar to the $N$. melanospilus 
species group distribution pattern. A phylogeographic pattern of $A$. bellottii demonstrates repeated vicariance and dispersal events resulting in broad coexistence of major haplogroups across major river basins [48]. Lowland coastal areas of East Africa also commonly experienced repeated Quaternary marine regression and transgressions due to sea level changes [49] and climatic variability $[50,51]$, including recent connection between island of Zanzibar (where $N$. melanospilus is also present) and mainland [17].

We propose that distribution of $N$. melanospilus lineages was shaped by repeated marine regression and transgressions in the Quaternary [49-51], transverse faulting in the area that pertains to Holocene [55] as well as repeated rainfall pattern changes that led to switches between woodland savanna and semi-deciduous forest habitats in lowland areas of coastal Tanzania [53]. While such repeated fluctuations led to coexistence of formerly more restricted lineages in coastal areas, other lineages were left more isolated and might have evolved into evolutionary independent units. This situation is reminiscent to the population genetic structure of a tigerfish, Hydrocynus tanzaniae, with lineages of Middle Pleistocene-dated divergence between the Ruvu and Rufiji basins [54], as well as to examples from other continents, such as intraspecific divergences of a freshwater goby, Rhinogobius duospilus, in Hong Kong streams and Iberian cyprinid, Squalius valentinus, whose limited dispersal capabilities resulted in a clear intra-specific signature of Quaternary climatic oscillations $[55,56]$.

Nothobranchius fishes typically live in ephemeral pools [16]. However, across extensive range of the genus their habitats vary greatly in their size, connectivity and inundation patterns. Nothobranchius populations are finely structured in small, short-existing pools in dry inland region of southern Mozambique [14, 42] where main river channels form significant barriers to dispersal and lead to allopatric species and strong intra-specific diversification [15]. In contrast, humid equatorial region appears to enable greater dispersal across river channels and between river basins ([5, 10, 44], present study), with Nothobranchius fishes occurring in extensive marshes and semipermanent streams $[5,16,57]$. Nothobranchius fishes are also present in the elevated part of equatorial East Africa ( $>800$ masl) and it remains to be tested how local populations are structured there. In that region, local topography and geographic history do not support as frequent dispersal as in coastal equatorial regions [10], while precipitation totals and existence of two rainy seasons differ from dry subtropical part of the genus distribution.

\section{Conclusions}

Distribution of genetic lineages of annual fishes from a wet part of the genus distribution (tropical lowland) appears not to be constrained by dispersal limits posed by main river channels and closely related lineages frequently coexist in secondary contact zones. Annual fishes are promising research system for understanding links between ecological and evolutionary processes $[4,58]$ and research on their interspecific and intraspecific diversification promises to shed more light on complex issues of African biogeography $[51,59,60]$.

\section{Supplementary information}

Supplementary information accompanies this paper at https://doi.org/10. 1186/s12862-019-1549-2.

Additional file 1: Protocols for genotyping of microsatellites and mitochondrial DNA. Table S1. Used microsatellite loci. Table S2. Primer sequences and marker features in GRZ strain of $N$. furzeri

Additional file 2: Additional figures supplementing results. Figure S1. Bayesian reconstruction of mitochondrial phylogeny of the $\mathrm{N}$. melanospilus species complex based on 83 ingroup and 1 outgroup haplotypes of the $657 \mathrm{bp}$ fragment of mitochondrial gene COI, including identity of individual samples. Bayesian inference posterior probabilities from MrBayes 3.2.6 are shown for each node. Figure S2. Correlation between $\operatorname{Ln}$ (distance) and linearized pairwise $F_{\mathrm{ST}} \mathrm{ENA}$ values $\left(F_{\mathrm{ST}} /\left(1-F_{\mathrm{ST}}\right)\right)$ tested by the Mantel tests (1000 permutations) analysed in GENEPOP. Figure S3. Mismatch distribution for two widespread mtDNA lineages. Dashed lines connect observed values and solid lines show the expected distribution under a demographic expansion model

Additional file 3: Additional table supplementing results. Table S3. Pairwise $F_{\text {ST }}$ estimates based on 10 microsatellite loci

Additional file 4: Evaluation of 20 runs in STRUCTURE 2.3.4 (Hubisz et al. 2009) for each number of presumable clusters from $K=1$ to $K=10$. Figure S2. Likelihood $(\operatorname{In} \operatorname{Pr}(X \mid K))$ of models in STRUCTURE for increasing number of hypothetical populations (K). Figure S3. Estimation of the best $\mathrm{K}$ division using the $\Delta \mathrm{K}$ criterion according to Evanno et al. (2005). The values indicate relative increase of credibility depending on the number of $\mathrm{K}$.

\section{Abbreviations}

AR: Allelic richness; COI: The mitochondrial gene of cytochrome oxidase subunit 1; FDR: The false discovery rate approach; HWE: Hardy-Weinberg equilibrium

\section{Acknowledgements}

We are grateful to Dr. Nazael Madalla for facilitating research plan and permits administration and David Alila for field assistance. We thank Eva Holánová for laboratory analysis.

\section{Authors' contributions}

MR conceived the idea; MR, BN, MP and RB collected the material, HL organised research agenda, VB analysed genetic data, VB and MR wrote the first version of the manuscript that was commented and approved by all authors.

\section{Funding}

Funding came through Czech Science Foundation (19-01781S) to MR.

\section{Availability of data and materials}

The datasets supporting the conclusions of this article are included within the article and its Additional files 1, 2, 3, 4. Microsatellite data were deposited to Figshare (doi: https://doi.org/10.6084/m9.figshare.9631907). New

sequences used in this study are available in GenBank (accession numbers MN413245-MN413327).

Ethics approval and consent to participate

Sampling complied with the legal regulations of the respective countries (research permit: RPGS/R/AS/11/2017; export permit AS/A/1; ethical approval: ref. 163-12). Samples utilised in the study have been lawfully acquired and 
were collected prior to The Nagoya Protocol on Access to Genetic Resources and the Fair and Equitable Sharing of Benefits Arising from Their Utilization to the Convention on Biological Diversity has been ratified in Tanzania. Except for voucher specimens, fish were not killed for the purpose of this study - sampling was performed by clipping a small piece of caudal fin that has rapid regenerating capability. Voucher specimens were killed by overdose of anaesthetics (clove oil), following approved protocol (ref. 163-12).

\section{Consent for publication}

Not applicable.

\section{Competing interests}

The authors declare that they have no competing interests.

\section{Author details}

${ }^{1}$ Czech Academy of Sciences, Institute of Vertebrate Biology, Květná 8, 60365 Brno, Czech Republic. ${ }^{2}$ Fontainebleau, France. ${ }^{3}$ Department of Animal, Aquaculture and Range Sciences, Sokoine University of Agriculture, P.O. Box 3004, Morogoro, Tanzania.

Received: 26 August 2019 Accepted: 27 November 2019

Published online: 06 January 2020

\section{References}

1. Fields PD, Reisser C, Dukić M, Haag CR, Ebert D. Genes mirror geography in Daphnia magna. Mol Ecol. 2015;24:4521-36.

2. Copilaş-Ciocianu D, Zimța AA, Grabowski M, Petrusek A. Survival in northern microrefugia in an endemic Carpathian gammarid (Crustacea: Amphipoda). Zool Scr. 2018:47:357-72

3. Williams DD. The biology of temporary waters. Oxford: Oxford University Press; 2006.

4. Berois N, García G, de Sá R. Annual fishes: life history strategy, diversity, and evolution. Boca Raton: CRC Press; 2015.

5. Watters BR. The ecology and distribution of Nothobranchius fishes. J Am Killifish Assoc. 2009;42:37-76.

6. Podrabsky JE, Riggs CL, Romney AL, Woll SC, Wagner JT, Culpepper KM, Cleaver TG. Embryonic development of the annual killifish Austrofundulus limnaeus: an emerging model for ecological and evolutionary developmental biology research and instruction. Dev Dyn. 2017;246:779-801.

7. Reichard M, Polačik M. Nothobranchius furzeri, an 'instant' fish from an ephemeral habitat. eLife. 2019;8:e41548.

8. Furness Al, Reznick DN, Springer MS, Meredith RW. Convergent evolution of alternative developmental trajectories associated with diapause in African and south American killifish. Proc Royal Soc London B. 2015;282:20142189.

9. Furness Al. The evolution of an annual life cycle in killifish: adaptation to ephemeral aquatic environments through embryonic diapause. Biol Rev. 2016:91:796-812.

10. Dorn A, Musilová Z, Platzer M, Reichwald K, Cellerino A. The strange case of east African annual fish: aridification correlates with diversification for a savannah aquatic group? BMC Evol Biol. 2014;14:210.

11. Nagy B, Cotterill FP, Bellstedt DU. Nothobranchius sainthousei, a new species of annual killifish from the Luapula River drainage in northern Zambia (Teleostei: Cyprinodontiformes). Ichthyol Explor Freshw. 2016;27:233-54.

12. Nagy B, Watters BR, van der Merwe PDW, Cotterill FPD, Bellstedt DU. Nothobranchius cooperi (Teleostei: Cyprinodontiformes): a new species of annual killifish from the Luapula River drainage, northern Zambia. Afr J Aquat Sci. 2017:42:201-18.

13. Dorn A, Ng'oma E, Janko K, Reichwald K, Polačik M, Platzer M, Cellerino A, Reichard M. Phylogeny, genetic variability and colour polymorphism of an emerging animal model: the short-lived annual Nothobranchius fishes from southern Mozambique. Mol Phylogenet Evol. 2011;61:739-49.

14. Bartáková V, Reichard M, Janko K, Polačik M, Blažek R, Reichwald K, Cellerino A, Bryja J. Strong population genetic structuring in an annual fish, Nothobranchius furzeri, suggests multiple savannah refugia in southern Mozambique. BMC Evol Biol. 2013;13:196.

15. Bartáková V, Reichard M, Blažek R, Polačik M, Bryja J. Terrestrial fishes: rivers are barriers to gene flow in annual fishes from the African savanna. J Biogeogr. 2015;42:1832-44.

16. Wildekamp RH. A world of Killies: atlas of the oviparous Cyprinodontiform fishes of the world, vol. 4. Elyria: American Killifish Association; 2004.
17. Costa WJEM. Two new species of seasonal killifishes of the Nothobranchius melanospilus species complex from the East Africa biodiversity hotspot (Cyprinodontiformes: Aplocheilidae). Vert Zool. 2019;69:73-82.

18. Myers N, Mittermeier RA, Mittermeier CG, Da Fonseca GA, Kent J. Biodiversity hotspots for conservation priorities. Nature. 2000;403:853.

19. Azeria ET, Sanmartín I, Ås S, Carlson A, Burgess N. Biogeographic patterns of the east African coastal forest vertebrate fauna. Biodivers Conserv. 2007;16: 883-912.

20. Wildekamp RH, Shidlovskiy KM, Watters BR. Systematics of the Nothobranchius melanospilus species group (Cyprinodontiformes: Nothobranchiidae) with description of two new species from Tanzania and Mozambique. Ichthyol Explor Freshw. 2009;20:237.

21. Costa WJEM. Taxonomic revision of the seasonal killifish genus Nothobranchius from Zanzibar, East Africa (Cyprinodontoidei: Aplocheilidae). J Nat Hist. 2017;51:1609-24.

22. Lanfear R, Frandsen PB, Wright AM, Senfeld T, Calcott B. PartitionFinder 2: new methods for selecting partitioned models of evolution for molecular and morphological phylogenetic analyses. Mol Biol Evol. 2016;34:772-3.

23. Ronquist $F$, Teslenko $M$, van der Mark $P$, Ayres DL, Darling A, Höhna $S$, Larget B, Liu L, Suchard MA, Huelsenbeck JP. MrBayes 3.2: efficient Bayesian phylogenetic inference and model choice across a large model space. Syst Biol. 2012;61:539-42

24. Miller MA, Pfeiffer W, Schwart T. Creating the CIPRES Science Gateway for inference of large phylogenetic trees. In: Proceedings of the Gateway Computing Environments Workshop (GCE). New Orleans: IEEE; 2010. p. 1-8.

25. Librado P, Rozas J. DnaSP v5: a software for comprehensive analysis of DNA polymorphism data. Bioinformatics. 2009;25:1451-2.

26. Fahey AL, Ricklefs RE, DeWoody JA. DNAbased approaches for evaluating historical demography in terrestrial vertebrates. Biol J Linn Soc. 2014;112: 367-86.

27. Schneider S, Excoffier L. Estimation of past demographic parameters from the distribution of pairwise differences when the mutation rates vary among sites: application to human mitochondrial DNA. Genetics. 1999;152:1079-89.

28. Rogers A. Genetic evidence for a Pleistocene population explosion. Evolution. 1995;49:608-15.

29. Excoffier L, Laval G, Schneider S. ARLEQUIN ver. 3.0: an integrated software package for population genetics data analysis. Evol Bioinform. 2005;1:47-50.

30. Chapuis M-P, Estoup A. Microsatellite null alleles and estimation of population differentiation. Mol Biol Evol. 2007;24:621-31.

31. Raymond M, Rousset F. GENEPOP (version 1.2): population genetics software for exact tests and ecumenicism. J Hered. 1995;86:248-9.

32. Rousset F. genepop'007: a complete re-implementation of the genepop software for windows and Linux. Mol Ecol Resour. 2008;8:103-6.

33. Storey JD. A direct approach to false discovery rates. J R Stat Soc Series B. 2002;64:479-98

34. Belkhir K, Borsa P, Chikhi L, Raufaste N, Bonhomme F. GENETIX 4.05, logiciel sous Windows TM pour la génétique des populations. In: Laboratoire Génome, Populations, Interactions, CNRS UMR; 1996-2004. p. 5000.

35. Goudet J. FSTAT, a program to estimate and test gene diversities and fixation indices (version 2.9.3). Available at https://kimura.univ-montp2.fr/ genetix. Accessed 2 Dec 2019.

36. Hubisz MJ, Falush D, Stephens M, Pritchard JK. Inferring weak population structure with the assistance of sample group information. Mol Ecol Res. 2009;9:1322-32.

37. Kopelman NM, Mayzel J, Jakobsson M, Rosenberg NA, Mayrose I. Clumpak: a program for identifying clustering modes and packaging population structure inferences across K. Mol Ecol Resour. 2015;15:1179-91.

38. Evanno G, Regnaut S, Goudet J. Detecting the number of clusters of individuals using the software structure: a simulation study. Mol Ecol. 2005;14:2611-20.

39. Wildekamp RW. Back to the theme Nothobranchius guentheri and Nothobranchius melanospilus, with additional notes to the N. melanospilus complex sensu Costa 2019. JAKA. 2019;52:43-52.

40. Vrtílek M, Reichard M. Patterns of morphological variation among populations of the widespread annual killifish Nothobranchius orthonotus are independent of genetic divergence and biogeography. J Zool Syst Evol Res. 2016:54:289-98.

41. Reichard M. The evolutionary ecology of African annual fishes. In: Berois N, García G, de Sá R, editors. Annual Fishes: Life History Strategy, Diversity, and Evolution: CRC Press; 2015. p. 133-58.

42. Vrtílek M, Žák J, Polačik M, Blažek R, Reichard M. Longitudinal demographic study of wild populations of African annual killifish. Sci Rep. 2018a;8:4774. 
43. Vrtílek M, Žák J, Pšenička M, Reichard M. Extremely rapid maturation of a wild African annual fish. Curr Biol. 2018b;28:R822-4.

44. Lambert JW, Reichard M, Pincheira-Donoso D. Live fast, diversify nonadaptively: evolutionary diversification of exceptionally short-lived annual killifishes. BMC Evol Biol. 2019;19:10.

45. de León JLP, León G, Rodríguez R, Metcalfe CJ, Hernández D, Casane D, García-Machado E. Phylogeography of Cuban Rivulus: evidence for allopatric speciation and secondary dispersal across a marine barrier. Mol Phylogenet Evol. 2014;79:404-14.

46. Duvernell DD, Meier SL, Schaefer JF, Kreiser BR. Contrasting phylogeographic histories between broadly sympatric topminnows in the Fundulus notatus species complex. Mol Phylogenet Evol. 2013;69:653-63.

47. Adams SM, Lindmeier JB, Duvernell DD. Microsatellite analysis of the phylogeography, Pleistocene history and secondary contact hypotheses for the killifish, Fundulus heteroclitus. Mol Ecol. 2006:15:1109-23.

48. García G, Gutiérrez V, Vergara J, Calvino P, Duarte A, Loureiro M. Patterns of population differentiation in annual killifishes from the Paraná-Uruguay-La Plata Basin: the role of vicariance and dispersal. J Biogeogr. 2012;39:1707-19.

49. Dutton A, Lambeck K. Ice volume and sea level during the last interglacial. Science. 2012;337:216-9.

50. Cohen AS, Stone JR, Beuning KRM, Park LE, Reinthal PZ, Dettman D, Scholz CA, Johnson TC, King JW, Talbot MR, Brown ET, Ivory SJ. Ecological consequences of early late Pleistocene megadroughts in tropical Africa. Proc Natl Acad Sci U S A. 2007;104:16422-7.

51. Lorenzen ED, Heller R, Siegismund HR. Comparative phylogeography of African savannah ungulates. Mol Ecol. 2012;21:3656-70.

52. Le Gall B, Gernigon L, Rolet J, Ebinger C, Gloaguen R, Nilsen O, Dypvig H, Deffontaines B, Mruma A. Neogene-Holocene rift propagation in Central Tanzania: Morphostructural and aeromagnetic evidence from the Kilombero area. GSA Bull. 2004;116:490-5 10.

53. Vincens A, Garcin Y, Buchet G. Influence of rainfall seasonality on African lowland vegetation during the Late Quaternary: pollen evidence from Lake Masoko, Tanzania. J Biogeogr. 2007;34:1274-88.

54. Goodier SA, Cotterill FP, O'Ryan C, Skelton PH, de Wit MJ. Cryptic diversity of African tigerfish (genus Hydrocynus) reveals palaeogeographic signatures of linked Neogene geotectonic events. PLoS One. 2011;6:e28775.

55. Wu TH, Tsang LM, Chen I-S, Chu KH. Multilocus approach reveals cryptic lineages in the goby Rhinogobius duospilus in Hong Kong streams: role of paleodrainage systems in shaping marked population differentiation in a city. Mol Phyl Evol. 2016;104:112-22.

56. Perea S, Doadrio I. Phylogeography, historical demography and habitat suitability modelling of freshwater fishes inhabiting seasonally fluctuating Mediterranean river systems: a case study using the Iberian cyprinid Squalius valentinus. Mol Ecol. 2015;24:3706-22.

57. Tweddle D, van der Waal BCW, Peel RA. Distribution and migration of the Caprivi killifish Nothobranchius caprivensis Watters, Wildekamp, Shidlovskiy 2015, an assessment of its conservation status and a note of other killifish in the same area. J Am Killifish Assoc. 2014:47:134-51.

58. Cellerino A, Valenzano DR, Reichard M. From the bush to the bench: the annual Nothobranchius fishes as a new model system in biology. Biol Rev. 2016;91:511-33.

59. Giddelo CS, Arndt AD, Volckaert FAM. Impact of rifting and hydrography on the genetic structure of Clarias gariepinus in eastern Africa. J Fish Biol. 2002; 60:1252-66.

60. Kaaya LT. Towards a classification of Tanzanian rivers: a bioassessment and ecological management tool. A case study of the Pangani, Rufiji and WamiRuvu river basins. Afr. J Aquat Sci. 2015;40:37-45.

\section{Publisher's Note}

Springer Nature remains neutral with regard to jurisdictional claims in published maps and institutional affiliations.

Ready to submit your research? Choose BMC and benefit from:

- fast, convenient online submission

- thorough peer review by experienced researchers in your field

- rapid publication on acceptance

- support for research data, including large and complex data types

- gold Open Access which fosters wider collaboration and increased citations

- maximum visibility for your research: over $100 \mathrm{M}$ website views per year

At BMC, research is always in progress.

Learn more biomedcentral.com/submissions 